\title{
Complete Genome Sequence Resource for the Necrotrophic Plant-Pathogenic Bacterium Pectobacterium carotovorum WPP14
}

\author{
Yingyu Liu, ${ }^{1}$ Tyler C. Helmann, ${ }^{2}$ Paul Stodghill, ${ }^{1,2}$ and Melanie J. Filiatrault ${ }^{1,2, \dagger}$ \\ ${ }^{1}$ School of Integrative Plant Science, Section of Plant Pathology and Plant-Microbe Biology, Cornell \\ University, Ithaca, NY 14853, U.S.A. \\ ${ }^{2}$ Emerging Pests and Pathogens Research Unit, Robert W. Holley Center for Agriculture and Health, \\ Agricultural Research Service, United States Department of Agriculture, Ithaca, NY 14853, U.S.A.
}

\begin{abstract}
Pectobacterium spp. are a major cause of loss in vegetable and ornamental plant production. One of these species, Pectobacterium carotovorum, can cause soft rot disease on many plants, particularly potato. These diseases lead to significant economic loss and pose food security threats by reducing crop yields in the field, in transit, and during storage. The Gramnegative enterobacterium $P$. carotovorum WPP14 is a particularly virulent strain for which there is no available closed genome, limiting the molecular research for this important pathogen. Here, we report a high-quality complete and annotated genome sequence of $P$. carotovorum WPP14. The 4,892,225-bp genome was assembled with Nanopore reads and polished with Illumina reads, yielding $394 \times$ and $164 \times$ coverage, respectively. This closed genome provides a resource for research on improved detection and biology of $P$. carotovorum, which could translate into improved disease management.
\end{abstract}

Pectobacterium spp. are necrotrophic bacterial pathogens that cause a wide range of diseases such as soft rot, stem wilt, and blackleg on a variety of plant hosts all over the world (Charkowski 2018; Kim and Jeon 2016; Lu et al. 2020; Voronina et al. 2019; Zlatković et al. 2019). Many species belong to the family Pectobacteriaceae. The species Pectobacterium carotovorum is divided into several subspecies. Recent genome analyses and taxonomic reassessments have resulted in some $P$. carotovorum strains of the subspecies being elevated to species rank and the designation of novel species of Pectobacterium (Oulghazi et al. 2019; Portier et al. 2019).

P. carotovorum WPP14 has been reported as one of the most virulent among different $P$. carotovorum (formerly Erwinia carotovora subsp. carotovora) strains when inoculated onto potato leaf petioles (Yap et al. 2004). A draft genome of $P$. carotovorum WPP14 is available with 731 contigs and 23.3-fold coverage (Glasner et al. 2008). Here, we report a de novo assembled, closed, and annotated genome sequence of $P$. carotovorum WPP14, which was originally isolated from diseased potato tubers and stems from Wisconsin during summer 2001 (Yap et al. 2004). This improved genome resource of $P$. carotovorum WPP14 could aid in population studies of Pectobacterium, advance understanding of pathogenicity, and facilitate the development of diagnostics tools of these widespread and destructive plant pathogens.

${ }^{\dagger}$ Corresponding author: M. J. Filiatrault; melanie.filiatrault@usda.gov

Mention of trade names or commercial products in this publication is solely for the purpose of providing specific information and does not imply recommendation or endorsement by the U.S. Department of Agriculture. USDA is an equal opportunity provider and employer.

The author(s) declare no conflict of interest.

Accepted for publication 21 July 2020.

\section{Funding}

This project is funded by the National Institute for Food and

Agriculture-Specialty Crops Research Initiative (2017-51181-26827). The funders had no role in the design of the study; in the collection, analyses, or interpretation of data; in the writing of the manuscript, or in the decision to publish the results.

\section{Keywords}

de novo assembly, Pectobacterium, soft rot, whole-genome sequencing 
Table 1. Genome summary and average nucleotide comparisons of Pectobacterium carotovorum WPP14 to other Pectobacterium spp. type strains

\begin{tabular}{|c|c|c|c|c|}
\hline Pectobacterium strains & Source & ANI $(\%)^{a}$ & Genome size (bp) & Contigs \\
\hline P. carotovorum WPP14 (this work) & NCBI: CP051652 & NA & $4,892,225$ & 1 \\
\hline P. carotovorum WPP14 (previous) & NCBI: ASM17315v1 & 99.87 & $4,823,187$ & 741 \\
\hline P. carotovorum ICMP 5702 & NCBI: ASM103905v1 & 97.50 & $4,774,457$ & 46 \\
\hline P. versatile CFBP 6051 & NCBI: ASM429668v1 & 95.87 & $4,949,375$ & 148 \\
\hline P. odoriferum NCPPB 3839 & NCBI: ASM75476v1 & 95.27 & $5,066,211$ & 156 \\
\hline P. aquaticum A212-S19-A16 & NCBI: ASM338256v2 & 94.04 & $4,348,076$ & 158 \\
\hline P. polaris NIBIO1006 & NCBI: ASM230735v1 & 93.58 & $4,826,824$ & 1 \\
\hline P. actinidiae $\mathrm{KKH} 3$ & NCBI: ASM80331v1 & 93.23 & $4,922,167$ & 3 \\
\hline P. brasiliense LMG21371 & NCBI: ASM75469v1 & 93.20 & $4,831,535$ & 109 \\
\hline P. peruviense IFB5232 & NCBI: ASM284734v1 & 89.85 & $4,785,880$ & 60 \\
\hline P. atrosepticum ICMP 1526 & NCBI: ASM103868v1 & 89.80 & $4,869,384$ & 33 \\
\hline P. betavasculorum NCPPB 2795 & NCBI: ASM74984v1 & 89.55 & $4,685,213$ & 93 \\
\hline P. zantedeschiae 9M & NCBI: ASM413779v1 & 89.29 & $5,054,916$ & 18 \\
\hline P. punjabense SS95 & NCBI: ASM302839v1 & 89.10 & $4,732,531$ & 73 \\
\hline P. wasabiae CFBP3304 & NCBI: ASM174218v1 & 89.00 & $5,043,228$ & 1 \\
\hline P. parmentieri RNS08-42-1A & NCBI: ASM174214v1 & 88.83 & $5,030,841$ & 1 \\
\hline P. fontis M022 & NCBI: ASM80321v1 & 88.50 & $4,151,564$ & 105 \\
\hline
\end{tabular}

${ }^{a}$ Average nucleotide identity (percent ANI) between the $P$. carotovorum WPP14 strain sequenced in this study compared with other type strains of Pectobacterium spp.

P. carotovorum WPP14 was cultured in Luria-Bertani medium at $28^{\circ} \mathrm{C}(10 \mathrm{~g}$ of tryptone, $5 \mathrm{~g}$ of yeast extract, and $10 \mathrm{~g} \mathrm{NaCl}$ per 1 liter of medium) (Bertani 1951). High molecular weight DNA was extracted from overnight bacterial cultures using a previously described method (Miller et al. 1988). DNA quantity was assessed using a NanoDrop 1000 Spectrophotometer (Thermo Fisher Scientific, U.S.A.) and a Qubit 3.0 Fluorometer (Thermo Fisher Scientific). DNA integrity was assessed using the Agilent 2100 BioAnalyzer system (Agilent, U.S.A.).

A complete scaffold of the genome was obtained using long reads from Oxford Nanopore Technologies (U.K.). Illumina (U.S.A.) short reads were used to polish the assembled genome. All software systems were run with their default settings unless otherwise noted. For long-read sequencing, the DNA library was prepared using the Nanopore Rapid Sequencing Kit SQK-RAD004 on a R9.4.1 SpotON flow cell with a MinION Mk1B device. This generated 242,453 reads with a total length of $1,987,696,323 \mathrm{bp}$ and a genome coverage of $394 \times$. The reads were base called using Guppy v3.5.1 and assembled using Flye v2.5 (Kolmogorov et al. 2019). For short-read sequencing, the DNA library was prepared using the NEBNext Ultra II FS DNA Library Prep Kit for Illumina with the NEBNext Multiplex Oligos (New England Biolabs, U.S.A.). AMPure XP beads (Beckman Coulter, U.S.A.) were used for DNA size selection and purification. The DNA library was sequenced on a MiSeq instrument (Illumina, U.S.A.) with the $2 \times 250$-bp mode, yielding 2,812,428 paired-end reads. Raw reads were processed to remove the adapter and low-quality sequences using Trimmomatic v0.39 (Bolger et al. 2014) (quality value $\leq 20$ ), ILLUMINACLIP:NEBNext.fa:2:30:10 LEADING:3 TRAILING:3 SLIDINGWINDOW:4:20 MINLEN:50). Duplicate read pairs were removed using a customized Perl script (https://github.com/Sunhh/NGS_data_processing/blob/master/ drop_dup_both_end.pl) (Sun et al. 2017). BWA v0.7.17 (Li and Durbin 2009) was used to align the resulting 2,535,566 high-quality cleaned Illumina read pairs to the draft genome, with a genome coverage of $164 \times$. Pilon v1.22 (Walker et al. 2014) was used for four rounds to correct the draft genome and yield the final polished genome. Circlator v1.5.5 was used with the "fix start" argument to set the start of the genome at the $d n a A$ gene (Hunt et al. 2015).

The final polished $P$. carotovorum WPP14 genome is 4,892,225 bp long with $52.07 \%$ GC content. It was annotated using the NCBI Prokaryotic Genome Annotation Pipeline (PGAP v4.11) (Tatusova et al. 2016). The resulting annotation contains 4,380 genes in total, with 4,201 protein-coding genes; 72 pseudogenes; 8, 7, and 7 ribosomal RNA (rRNA)-coding sequences (5S, 16S, and 23S rRNAs), 76 tRNAs, and 9 noncoding RNAs; and 5 clustered regularly interspaced short palindromic repeat (CRISPR) arrays. Whole-genome comparison of $P$. carotovorum WPP14 and other Pectobacterium type strains based on average nucleotide identity (ANI) can be found in Table 1, including their genome sizes and number of contigs. Genome sequences were retrieved from publicly available sources (Glasner et al. 
2008; NCBI Research Coordinators 2016). ANI was calculated using FastANI (Jain et al. 2018). Based on ANI, P. carotovorum WPP14 showed the closest similarity to the P. carotovorum type strain ICMP 5702.

Data availability. The $P$. carotovorum WPP14 genome sequence has been deposited at NCBI GenBank under accession number CP051652. The Nanopore and Illumina raw reads have been deposited in the Sequence Read Archive under accession numbers SRR11435198 and SRR11435197, respectively.

\section{Acknowledgments}

We thank the Genomics Facility of the Biotechnology Resource Center at the Cornell University's Institute of Biotechnology for their help with sequencing experiments.

\section{Literature Cited}

Bertani, G. 1951. Studies on lysogenesis. I. The mode of phage liberation by lysogenic Escherichia coli. J. Bacteriol. 62:293-300.

Bolger, A. M., Lohse, M., and Usadel, B. 2014. Trimmomatic: A flexible trimmer for Illumina sequence data. Bioinformatics 30:2114-2120.

Charkowski, A. O. 2018. The changing face of bacterial soft-rot diseases. Annu. Rev. Phytopathol. 56:269-288.

Glasner, J. D., Marquez-Villavicencio, M., Kim, H. S., Jahn, C. E., Ma, B., Biehl, B. S., Rissman, A. I., Mole, B., Yi, X., Yang, C. H., Dangl, J. L., Grant, S. R., Perna, N. T., and Charkowski, A. O. 2008. Niche-specificity and the variable fraction of the Pectobacterium pan-genome. Mol. Plant-Microbe Interact. 21:1549-1560.

Hunt, M., Silva, N. D., Otto, T. D., Parkhill, J., Keane, J. A., and Harris, S. R. 2015. Circlator: Automated circularization of genome assemblies using long sequencing reads. Genome Biol. 16:294.

Jain, C., Rodriguez-R, L. M., Phillippy, A. M., Konstantinidis, K. T., and Aluru, S. 2018. High throughput ANI analysis of $90 \mathrm{~K}$ prokaryotic genomes reveals clear species boundaries. Nat. Commun. 9:5114.

Kim, Y. S., and Jeon, Y. 2016. First report of Orostachys malacophyllus soft rot caused by Pectobacterium carotovorum subsp. carotovorum in Korea. Plant Dis. 100:208.

Kolmogorov, M., Yuan, J., Lin, Y., and Pevzner, P. A. 2019. Assembly of long, errorprone reads using repeat graphs. Nat. Biotechnol. 37:540-546.

$\mathrm{Li}, \mathrm{H}$., and Durbin, R. 2009. Fast and accurate short read alignment with Burrows-Wheeler transform. Bioinformatics 25:1754-1760.

Lu, Q., Liu, Y., Yan, F., Zhu, L., and Liu, P. 2020. First report of Pectobacterium carotovorum subsp. actinidiae causing summer canker on kiwifruit in Zhejiang province of China. Plant Dis. 104:561.

Miller, S. A., Dykes, D. D., and Polesky, H. F. 1988. A simple salting out procedure for extracting DNA from human nucleated cells. Nucleic Acids Res. 16:1215.

NCBI Research Coordinators. 2016. Database resources of the National Center for Biotechnology Information. Nucleic Acids Res. 44:D7-D19.

Oulghazi, S., Cigna, J., Lau, Y. Y., Moumni, M., Chan, K. G., and Faure, D. 2019. Transfer of the waterfall source isolate Pectobacterium carotovorum M022 to
Pectobacterium fontis sp. nov., a deep-branching species within the genus Pectobacterium. Int. J. Syst. Evol. Microbiol. 69:470-475.

Portier, P., Pédron, J., Taghouti, G., Fischer-Le Saux, M., Caullireau, E., Bertrand, C., Laurent, A., Chawki, K., Oulgazi, S., Moumni, M., Andrivon, D., Dutrieux, C., Faure, D., Hélias, V., and Barny, M.-A. 2019. Elevation of Pectobacterium carotovorum subsp. odoriferum to species level as Pectobacterium odoriferum sp. nov., proposal of Pectobacterium brasiliense sp. nov. and Pectobacterium actinidiae sp. nov., emended description of Pectobacterium carotovorum and description of Pectobacterium versatile sp. nov., isolated from streams and symptoms on diverse plants. Int. J. Syst. Evol. Microbiol. 69: 3207-3216.

Sun, H., Wu, S., Zhang, G., Jiao, C., Guo, S., Ren, Y., Zhang, J., Zhang, H., Gong, G., Jia, Z., Zhang, F., Tian, J., Lucas, W. J., Doyle, J. J., Li, H., Fei, Z., and Xu, Y. 2017. Karyotype stability and unbiased fractionation in the paleo-allotetraploid Cucurbita genomes. Mol. Plant 10:1293-1306.

Tatusova, T., DiCuccio, M., Badretdin, A., Chetvernin, V., Nawrocki, E. P., Zaslavsky, L., Lomsadze, A., Pruitt, K. D., Borodovsky, M., and Ostell, J. 2016. NCBI prokaryotic genome annotation pipeline. Nucleic Acids Res. 44:6614-6624.

Voronina, M. V., Kabanova, A. P., Shneider, M. M., Korzhenkov, A. A., Toschakov, S. V., Miroshnikov, K. K., Miroshnikov, K. A., and Ignatov, A. N. 2019. First report of Pectobacterium carotovorum subsp. brasiliense causing blackleg and stem rot disease of potato in Russia. Plant Dis. 103:364.

Walker, B. J., Abeel, T., Shea, T., Priest, M., Abouelliel, A., Sakthikumar, S., Cuomo, C. A., Zeng, Q., Wortman, J., Young, S. K., and Earl, A. M. 2014. Pilon: An integrated tool for comprehensive microbial variant detection and genome assembly improvement. PLoS One 9:e112963.

Yap, M.-N., Barak, J. D., and Charkowski, A. O. 2004. Genomic diversity of Erwinia carotovora subsp. carotovora and its correlation with virulence. Appl. Environ. Microbiol. 70:3013-3023.

Zlatković, N., Prokić, A., Gašić, K., Kuzmanović, N., Ivanović, M., and Obradović, A. 2019. First report of Pectobacterium carotovorum subsp. brasiliense causing soft rot on squash and watermelon in Serbia. Plant Dis. 103: 2667 